\title{
Planeando para la vida en el Centro de Autoacceso: Reflexiones de una buena práctica
}

\author{
Planning for life at the Self-Access Center: Good Practice Reflections
}

\author{
Guillermo Huerta-Gutiérrez ${ }^{a}$, María G. Alcubilla-Hernández ${ }^{b}$
}

\begin{abstract}
:
The Self Access Centers have been characterized as a means to foster students' autonomy at the Universidad Veracruzana. The SelfAccess Center USBI Xalapa, has been a Self-access language learning center for 20 years. During this time, it has been well-known due to its wide range of learning resources to support students, such as learning objects, pathways, worksheets, interactive presentations, among others. These resources are used in a systematic way in our courses, English I and English II, through a working dynamic which starts with designing a studying plan. In this plan, all resources that appear in our pathways are integrated so as to support and guide our students' self-learning work. While working on this plan, not only do students foster their language skills, but also they develop metacognitive strategies such as planning, organization, time management, decision making, setting goals and needs analysis, nowadays considered soft skills for life and essential for students' integral formation. In this sense, the objective of this article is to share the results of this good practice and some students' reflection about it, this may serve as a reference for other selfaccess centers.
\end{abstract}

Keywords:

Autonomy, Self-access center, self-learning, soft skills, studying plan

\section{Resumen:}

Los Centros de Autoacceso se han caracterizado por ser un medio idóneo para fomentar la autonomía de los estudiantes en la Universidad Veracruzana. El Centro de Autoacceso USBI Xalapa, lleva 20 años como centro de aprendizaje de idiomas. En esta trayectoria se ha caracterizado por la gama de recursos con los que cuenta tales como objetos de aprendizaje, rutas y hojas de trabajo, presentaciones interactivas, entre otros. Estos recursos son trabajados de manera sistemática en los cursos de Inglés I y II a través de una dinámica que inicia con la elaboración de un plan de trabajo. En éste convergen de manera organizada los diferentes recursos que se encuentran en las rutas de trabajo, base del trabajo autónomo de nuestros estudiantes. Durante el trabajo en este plan, los estudiantes no sólo desarrollan sus habilidades del idioma, sino que también ponen en práctica múltiples habilidades metacognitivas, tales como la planificación y gestión del tiempo, toma de decisiones, establecimiento de metas, análisis de necesidades, actualmente consideradas habilidades blandas para la vida y esenciales en la formación integral de los estudiantes. Nuestro objetivo es compartir los resultados de esta buena práctica y algunas reflexiones de nuestros estudiantes para que pueda servir como punto de referencia a otros Centros de Autoacceso.

Palabras Clave:

Autoaprendizaje, autonomía, Centro de Autoacceso, habilidades blandas, plan de trabajo

\section{INTRODUCTION}

Desde hace más de dos décadas se ha venido fomentando la autonomía del aprendizaje en estudiantes universitarios gracias a la apertura de Centros de Autoacceso (CAA) en diversas instituciones de educación superior en todo el país. Autonomía es un concepto muy amplio, pero en el área de enseñanzaaprendizaje se relaciona con infinidad de palabras como

\footnotetext{
${ }^{a}$ Guillermo Huerta Gutiérrez, Universidad Veracruzana, México, https://orcid.org/0000-0002-2240-7886, Email: guihuerta@uv.mx

b María Guadalupe Alcubilla Hernández, Universidad Veracruzana, México, https://orcid.org/0000-0002-7977-8354, Email:
} galcubilla@uv.mx 
autoaprendizaje, auto-instrucción, aprendizaje independiente o aprendizaje semi-dirigido, entre otros. Precisamente, en este último concepto se sustenta el trabajo que se realiza en el Centro de Autoacceso USBI Xalapa desde 1999 cuando abrió sus puertas. Desde entonces, en este CAA se ha puesto en marcha una dinámica de trabajo que permite guiar e ir entrenando a nuestros estudiantes hacia la autonomía en su aprendizaje. Dicha dinámica de trabajo se apoya en diversas acciones, recursos y documentos que han ido evolucionando con base en las necesidades de nuestros estudiantes y objetivos institucionales. Una de estas metas es la formación integral de los estudiantes, es decir, prepararlos para la vida, para los requerimientos de nuestra sociedad actual y no solo en la formación académica de una profesión. El CAA USBI Xalapa contribuye a esta formación de habilidades para la vida en todos sus cursos en modalidad autónoma. Aquí hablaremos, específicamente, del trabajo que se ha realizado en los cursos o las Experiencias Educativas (EE) de Inglés I y II del Área de Formación Básica General (AFBG) donde los estudiantes realizan la planeación de su trabajo como parte de la dinámica de trabajo de sus cursos.

\section{CONTEXTO}

La Universidad Veracruzana (UV) es una institución de educación pública y autónoma situada en el estado de Veracruz. Es considerada una de las cinco más grandes del país ya que actualmente cuenta con una población superior a los 80.000 estudiantes distribuidos en cinco sedes regionales. Ofrece más de 300 programas educativos de nivel técnico, técnico superior universitario, licenciatura y posgrado cubriendo todas las áreas de conocimiento. (Universidad Veracruzana [UV], 2021)

Los Centros de Autoacceso (CAA) de la Universidad Veracruzana fueron concebidos como parte de un proyecto nacional entre la Secretaría de Educación Pública (SEP) y el Consejo Británico a principios de los noventa. El primer paso de este proyecto fue un entrenamiento a gran escala sobre el concepto de centros de autoacceso, su apertura e implementación en las instituciones interesadas. La UV fue una de las primeras cinco universidades que aperturaron un Centro de Autoacceso en México. Así nació el Centro de Autoacceso de la Facultad de Idiomas (CAAFI) en mayo de 1994.

En septiembre de 1999, la UV abrió nueve Centros de Autoacceso más distribuidos en las cinco sedes regionales. Su creación formó parte también del Nuevo Modelo Educativo (NME) basado en competencias que inició en ese mismo año en varios programas educativos. El principal objetivo de la creación de estos CAA fue promover el aprendizaje del inglés en modalidad autónoma en un nivel básico entre los estudiantes universitarios ofreciendo las experiencias educativas de Inglés I y 2 del Área de Formación Básica General (AFBG) del NME. Hoy en día, ya son 11 CAA con los que cuenta la UV y se encuentran abiertos a toda la comunidad universitaria y público en general que desee aprender idiomas en otras modalidades diferentes a la presencial o utilizarlos como centro de práctica para las clases presenciales. Se ofrecen cursos de otros idiomas, no solo inglés y en niveles más avanzados. (UV, 2021)

\section{CENTRO DE AUTOACCESO USBI XALAPA}

El Centro de Autoacceso USBI Xalapa abrió sus puertas el 1 de septiembre de 1999 y es el más grande de la UV. Este se encuentra ubicado en la Unidad de Servicios Bibliotecarios y de Información (USBI) en la Zona Universitaria de la ciudad capital, Xalapa, Veracruz. Este es un espacio educativo dedicado a la impartición de inglés y francés en modalidad autónoma, virtual y multimodal. Su objetivo es contribuir a la formación integral de los universitarios a través de formas innovadoras de enseñanza-aprendizaje de lenguas extranjeras, con un gran sentido de responsabilidad, respeto y promoción de las diferentes culturas, además de un alto nivel académico, que lo identifican dentro de la comunidad universitaria. (UV, 2021)

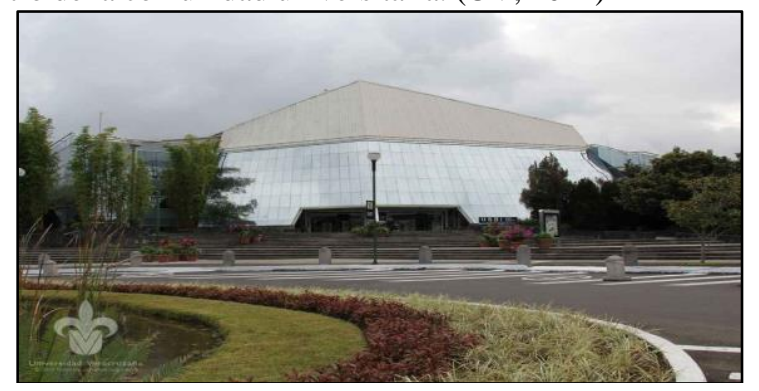

Imagen 1. USBI Xalapa. Universidad Veracruzana

Este CAA tiene capacidad para albergar alrededor de 340 usuarios simultáneamente organizados en las diferentes áreas: un área de audio, dos áreas de cómputo, una sala de lectura y escritura, dos salas de conversación, tres salas de asesorías, una sala de asesores, una sala de usos múltiples y la coordinación del CAA. Además, cuenta con la recepción y un área de anaqueles para materiales impresos y bibliográficos.

El CAA USBI Xalapa cuenta con personal académico calificado e incluye a siete asesores de inglés de tiempo completo, dos asesores de francés de tiempo completo, tres técnicos académicos, dos facilitadores académicos, un personal de confianza, uno de apoyo y la coordinadora del CAA. Este CAA ofrece actualmente los cursos de Inglés I y 2 del AFBG en modalidad autónoma y virtual, cursos de Francés básico e intermedio del Área de Formación de Elección Libre (AFEL) en modalidad autónoma, así como cursos de Inglés III(Nivel A2) básico e Inglés I intermedio (Transición del nivel A2 al B1) en modalidad multimodal. Además, este CAA es centro aplicador nacional de exámenes de certificación de lengua inglesa (EXAVER) y apoya diferentes acciones de la Dirección de Internacionalización de la UV relacionadas con la movilidad de estudiantes y académicos.

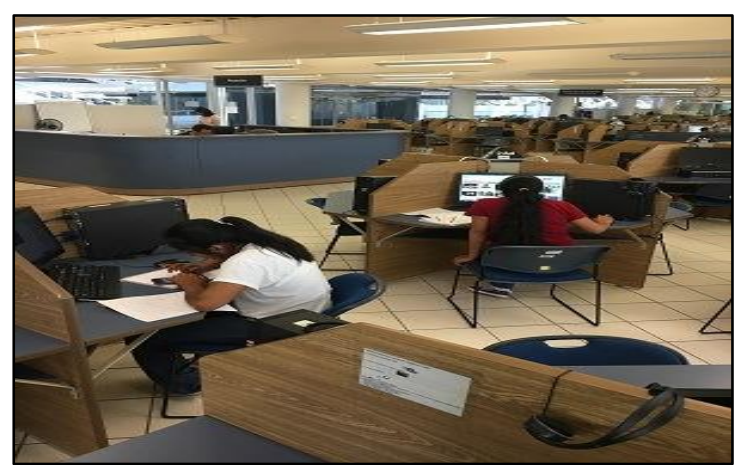

Imagen 2. Centro de Autoaccseo USBI Xalapa

El Área de formación está integrada por las EE: Literacidad Digital, Pensamiento Crítico, Lectura y Escritura e Inglés I e Inglés 2. Estos dos últimos, son cursos de Inglés Básico, 4 habilidades, para ser trabajados en tiempo aproximado de 90 
horas. Al completar estos dos cursos se espera que el estudiante alcance un nivel A1+ de acuerdo con el Marco Común Europeo de Referencia (MCER). Estos forman parte del programa de estudios de todas las carreras de licenciatura que ofrece la UV, tienen un valor de 6 créditos cada uno y son de carácter obligatorio. Los estudiantes tienen la opción de acreditar estas experiencias educativas de diversas formas, a través de un curso presencial, virtual o autónomo en un CAA, a través de un examen de competencias o presentando una certificación nacional como EXAVER o una internacional como los de la Universidad de Cambridge o TOEFL IBT. Cada semestre, este CAA atiende aproximadamente alrededor de 500 alumnos que cursan Inglés I y 400 que cursan Inglés II en modalidad autónoma.

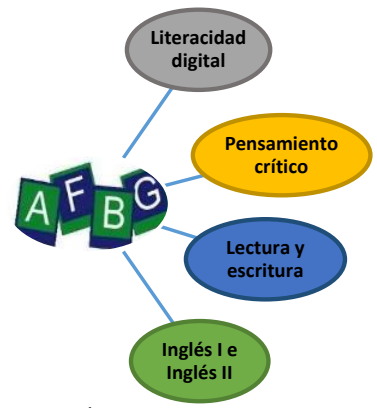

Figura 1. Área de Formación Básica General

El CAA USBI Xalapa también da servicio a estudiantes de clases presenciales para realizar sus prácticas. Sin embargo, este CAA es considerado más un centro de aprendizaje que un centro de práctica (Sturtridge, 1992), ya que la mayor parte de nuestros estudiantes realizan sus cursos completos aquí con la guía de los asesores y el apoyo de diferentes recursos y documentos tales como rutas de trabajo, asesorías, materiales impresos y digitales, guías de aprendizaje, autoevaluaciones y evaluaciones, talleres de conversación y de estrategias, actividades culturales, programas de apoyo, entre otros.

\section{AUTONOMÍA}

Autonomía es un concepto clave, base del trabajo que se realiza en el Centro de Autoacceso USBI Xalapa. De acuerdo con la Real Academia Española, autonomía es "la capacidad de los sujetos de derecho para establecer reglas de conducta para sí mismos y en sus relaciones con los demás dentro de los límites que la ley señala". Este término fue denominado como "a buzzword of the 1990's" (Little, 1991, p.2 en Benson y Voller, 1997, p.1) debido a la gran importancia que tuvo en el área de aprendizaje de idiomas en aquel momento. El término autonomía describe la situación en la cual el aprendiente es totalmente responsable de todas las decisiones concernientes a su aprendizaje, así como la implementación de esas decisiones. La autonomía total en el aprendizaje implica que "there is no involvement of a teacher or an institution and the learner is also independent of specially prepared materials". (Dickinson, 1987, p.11). Asimismo, existen términos que se relacionan o que están implícitos en este concepto amplio de autonomía, tales como autoaprendizaje, auto instrucción, trabajo independiente, entre otros, como se muestra en la figura 2.

Sin embargo, en nuestro contexto no se espera autonomía total en los alumnos ya que para la mayoría es la primera vez que se enfrenta a esta modalidad de aprendizaje en su trayectoria académica, por lo que podríamos hablar de semi-autonomía. Según Dickinson, semi-autonomía se refiere a "the stage at which learners are preparing for autonomy”. (1987, p.11).

Un sinónimo de autonomía es auto-dirección, que ha sido definido por Knowles como "a process in which individuals take the initiative, with or without the help of others, in diagnosing their learning needs, formulating learning goals, identifying human and material resources for learning, choosing and implementing appropriate learning strategies and evaluating their outcomes..." (1975, p.8 en Wenden y Rubin, 1987 p.11).

El término auto-instrucción es también utilizado como sinónimo. Este es un término neutral que se refiere a situaciones en general donde los aprendientes trabajan sin el control directo de un maestro (Dickinson, 1987). Otros autores como Sheerin se refiere a autonomía del aprendizaje como aprendizaje independiente y menciona que "involves learners taking responsibility for their own learning and developing effective learning strategies; in other words, learning how to learn". (s.f. p.3 en Benson y Voller, 1997 p.56).

El enfoque autónomo está centrado en el aprendiente, es decir, se enfoca en el aprendizaje más que en la enseñanza. Enfatiza "the role of learners as active agents in their own learning" (Benson y Voller, 1997 p.7). El aprendizaje autónomo de idiomas se ha asociado regularmente con el trabajo individualizado y la idea de que cada aprendiente tiene su propio estilo, capacidades y necesidades de aprendizaje.

Hay razones de toda índole para promover el aprendizaje independiente, pero todas emergen de una creencia general entre los maestros, no solo del área de idiomas, y es que el aprendizaje es más efectivo cuando los aprendientes juegan un papel más activo en su proceso de aprendizaje, asumiendo responsabilidades sobre su aprendizaje y participando en las decisiones que tienen que ver con este.

Autoaprendizaje es otro término similar y según Granados (2017) es un proceso por el cual aprendemos a aprender, a través del desarrollo de capacidades cognitivas como la atención, la memoria, la comprensión y la creatividad, entonces primero requerimos del acompañamiento de alguien que nos lleve a reconocer cómo aprendemos, en qué podemos mejorar y qué sería recomendable incorporar.

De acuerdo con Benson (2008), los términos, autoaprendizaje, aprendizaje independiente, aprendizaje autodirigido o auto instrucción, describen básicamente diversas formas y grados de aprender por sí mismo, mientras que la autonomía se refiere a habilidades y actitudes relacionadas con la capacidad de controlar el propio aprendizaje.

Llegar a convertirse en un aprendiente independiente es un proceso largo. "At the lowest level of independence a learner might be willing and able to work unsupervised but in every other respect be dependent on a teacher or advisor for direction". (Sheerin, s.f. p.5 en Benson y Voller, 1997 p.58). Podemos decir que en el Centro de Autoacceso USBI Xalapa los estudiantes realizan los cursos de Inglés del AFBG en modalidad autónoma siguiendo un proceso semi-dirigido, ya que tratamos de proveer a los usuarios con recursos que les permitan lograr sus objetivos 
como lo mencionan Skinner y Belmont (1993), en este caso, el objetivo es la autonomía en el aprendizaje.

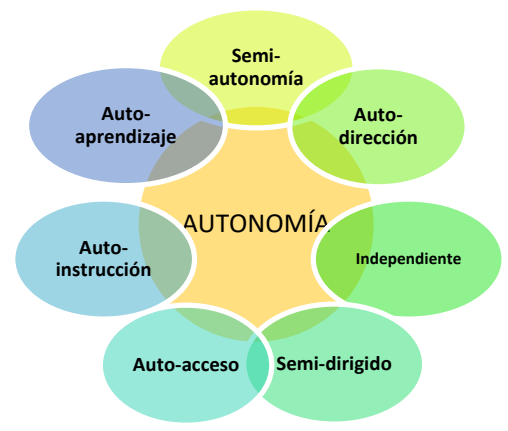

Figura 2. Autonomía y palabras relacionadas.

\section{APRENDIZAJE DE IDIOMAS EN UN CENTRO DE AUTOACCESO}

Autoacceso se refiere a "the organization of learning materials (and possibly equipment) to make them directly available to the learner" (Dickinson, 1987, p.10). De manera similar, Sturtridge define a un centro de autoacceso como "any system, which makes materials available to language learners so that they can choose to work freely, usually without a teacher or with very limited support" (1992, p.4). Con esta definición, podemos decir que aprender en un centro de autoacceso es una forma diferente de aprender, es auto-instrucción utilizando materiales y recursos disponibles en un solo lugar. La teoría detrás del aprendizaje en un centro de autoacceso es que los estudiantes aprenden mejor cuando se les da libertad para aprender de manera independiente. Así, ellos pueden escoger la forma de aprender que más les acomode, de acuerdo a su estilo de aprendizaje, los materiales que necesiten y les agraden y dedicarles el tiempo que ellos requieran.

Se puede decir que el aprendizaje en un centro de autoacceso es exitoso cuando permite a los estudiantes mejorar tanto sus habilidades, en este caso en el idioma que estén aprendiendo, así como sus estrategias de aprendizaje. Gremmo y Riley (1995) mencionan que en un centro de autoacceso, los estudiantes pueden escoger sus propias estrategias de aprendizaje no importando el nivel en el que se encuentren, la velocidad en la que trabajen o el contenido que estén trabajando. Lawson (2006), por otro lado, indica que en un centro de autoacceso, los estudiantes pueden seleccionar sus propias formas de aprender con el beneficio de contar con la ayuda y consejos de un maestro.

De acuerdo con Knowles (1975), el aprendizaje en un centro de autoacceso es un proceso en el cual los individuos tienen la responsabilidad de diagnosticar sus necesidades, establecer sus metas, planear su trabajo, identificar recursos, seleccionar estrategias de aprendizaje y evaluar su desempeño, con o sin la ayuda de otros.

Sturtridge (1992) describe cuatro tipos de centros de autoacceso de idiomas y sus funciones (Figura 3):

1. Centro de instrucción (The instruction center): Este puede incluir un laboratorio de idiomas o sala de cómputo, y ofrece a los estudiantes un programa planeado con anticipación que puede funcionar como sustituto del maestro o apoyo extra para clases presenciales.

2. Centro de práctica (The practice center): Se concentra solo en materiales y recursos de apoyo a contenidos vistos en clases presenciales. Su trabajo va de la mano con el programa de enseñanza institucional.

3. Centro de habilidades (The skill center): Se concentra solo en una habilidad. Los centros de lectura o de comprensión oral son los más comunes.

4. Centro de Aprendizaje (The learning center): Este permite ampliar el aprendizaje de idiomas. Cuenta con un suministro de materiales de todas las habilidades que van más allá de solo proveer práctica, consolidación o evaluación. Están creados para estudiantes que no acuden a clases presenciales de idiomas si no que desean estudiar por cuenta propia teniendo la guía de un asesor y apoyados con diferentes recursos.

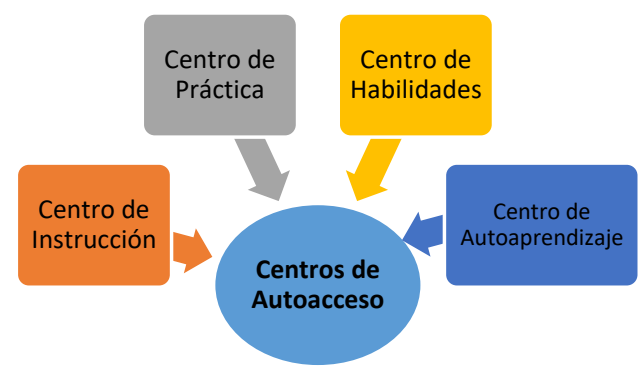

Figura 3. Tipos de Centros de Autoacceso de idiomas.

Nosotros en el Centro de Autoacceso USBI Xalapa, hemos tomado la definición de Sturtridge que se refiere a un centro de autoacceso como cualquier sistema que ponga los materiales a disposición de los estudiantes de lenguas de modo que ellos puedan elegir y trabajar libremente, por lo general sin un maestro o con un apoyo limitado, esto es, como un Centro de Aprendizaje de Idiomas.

\section{Dinámica de trabajo del CAA para los cursos autónomos}

Los centros de Autoacceso se caracterizan por promover el aprendizaje autónomo como lo señala Serra (2000) "self-access can be seen as one context in which autonomy can be developed." (p.97). En este sentido Doddis (1999) en su reseña de la obra de Gardner y Miller menciona que estos autores se refieren al término autoacceso para referirse "a un enfoque cuyo propósito final es estimular la autonomía” (p.287).

Para poder cumplir con el objetivo que se plantea para los Centros de Autoacceso es importante tener claras las actividades que se van a desarrollar debido a que "las actividades son, pues, el corazón del proceso de aprendizaje" (Alicia Peña Calvo, 1997, p. 623). En este sentido, en el Centro de Autoacceso USBI Xalapa se han planteado una serie de procedimientos que son presentados en diferentes actividades a los estudiantes. Para empezar, se han organizado una serie de asesorías que buscan sensibilizar a los usuarios sobre la dinámica de trabajo, las instalaciones, sobre su avance, retroalimentación y orientación en general. Por otro lado, se han desarrollado diferentes materiales y recursos como promotores 
del aprendizaje autónomo. En las siguientes líneas describiremos estos procedimientos, materiales y recursos.

\section{Procedimientos del trabajo autónomo}

Los procedimientos consisten en una serie de asesorías que se dan a lo largo del curso, desde que este inicia hasta que concluye. Mynard (2018) sugiere que "Advising (...) involves the process and practice of helping students to direct their own paths so as to become more effective and more autonomous language learners" (p.30). A continuación, se describen a detalle cada una de las asesorías que se ofrecen en el CAA USBI Xalapa:

a) Curso de Inducción: El trabajo inicia con el curso de inducción cuyo objetivo es dar a conocer la filosofía detrás de la autonomía del aprendizaje, así como sus espacios, personal, recursos, servicios, etc. Este tiene una duración de cuarenta minutos aproximadamente y se ofrece en las primeras dos semanas del semestre, consta de una parte teórica y otra práctica y es requisito hacerlo para poder inscribirse al CAA. Los estudiantes pueden tomar este curso de manera presencial o de manera virtual desde la página del centro.

b) Primera Asesoría: Su objetivo es explicar las características de los cursos, la dinámica del trabajo autónomo, el uso de recursos y documentos necesarios para llevar a la práctica su plan de trabajo. Además, se dan a conocer los lineamientos generales del curso. Aquí, se orienta a los estudiantes para que puedan realizar un análisis de necesidades basado en experiencias anteriores, al establecimiento de metas a corto plazo, así como a planear su trabajo. Esta asesoría se ofrece de manera presencial en pequeños grupos con una duración de una hora treinta minutos aproximadamente. Existe la opción de realizarla de manera virtual e individual en aproximadamente cuarenta minutos desde la página de nuestro centro.

Después de esta asesoría los estudiantes comienzan su trabajo autónomo, sin embargo, en las primeras secciones necesitarán del apoyo de todos los recursos, asesores y demás personal del CAA en lo que se van familiarizando con la dinámica de trabajo.

c) Asesoría para Reporte y Seguimiento: El reporte, como su nombre lo indica, se refiere a la actividad que llevan a cabo los estudiantes cuando acuden al CAA a reportar sus avances de secciones. Es una asesoría que se oferta durante todo el día en diferentes horarios, no se reserva y tiene una duración de entre cinco y diez minutos, dependiendo de las observaciones hechas por el asesor o de las inquietudes que el usuario pueda tener.

d) Asesoría de Revisión de Modular: Las evaluaciones modulares nos permiten tener un panorama general del avance y logro de los objetivos de aprendizaje de nuestros estudiantes. Estas se llevan a cabo al final de cada módulo (2 para Inglés I y 3 para Inglés II). Después de que el estudiante presenta estas evaluaciones deben agendar esta asesoría. Aquí, se revisan los resultados junto con el estudiante para poder dar una retroalimentación más directa y puedan reflexionar sobre sus debilidades y fortalezas.

Es importante recalcar que el papel que juega el asesor es determinante para estas asesorías ya que como mencionan Olea et al (2019) "El asesor debe dar las pautas para relacionar temas, elaborar las actividades, dar orientación didáctica para la comprensión y aplicación de los contenidos aprobados, realizar la evaluación y el seguimiento permanente del proceso de aprendizaje del alumno" (p.3)

\section{Materiales y recursos}

Muchos autores han señalado la relevancia e importancia de los materiales ya que "son la fuente principal de aprendizaje y están diseñados para que el estudiante pueda aprender de ellos y con ellos" (Alicia Peña Calvo, 1997, p. 623). Del mismo modo, autores como Jeremy Harmer (2007) conciben a los Centros de Autoacceso como una alternativa para el aprendizaje de idiomas en donde se puede tener acceso a una gran gama de materiales tales como libros de gramática, de trabajo, de referencia, diccionarios, de audio, de video, entre otros. El CAA USBI Xalapa no es la excepción y entre sus materiales y recursos se encuentran las rutas y hojas de trabajo, actividades de audio, presentaciones de PowerPoint interactivas, objetos de aprendizaje, autoevaluaciones, materiales de aprender a aprender entre otros.

\section{PLAN DE TRABAJO}

Para iniciar con la descripción del Plan de Trabajo, es importante definir aprendizaje. De acuerdo con Klimenko y Alvares, "el aprendizaje es un proceso complejo, que contiene unos elementos constituyentes, que depende de ciertos factores tanto internos como externos, requiere de medios externos e internos y cuenta con diferentes niveles de participación de la conciencia" (2009, p.15). No se aprende de una sola manera. Hay distintos tipos de aprendizaje que representan diferentes formas de actuar con la información que se aprende. La sociedad actual requiere un aprendizaje autónomo y estratégico de los individuos, esto es, un aprendizaje significativo, autorregulado, consciente que permite orientarse en la gran cantidad de la información disponible mediante un claro discernimiento basado en criterios sólidos y sustentados (Pozo et ál., 2006). Esta forma de aprender requiere, entonces, la toma de consciencia y la reflexión sobre las características, capacidades y habilidades particulares que facilitan o dificultan el proceso de aprendizaje, sobre las estrategias cognitivas que pueden ponerse en práctica, así como aprender a regular el propio proceso de aprendizaje monitoreándolo de una manera independiente.

Klimenko y Alvares (2009) afirman que un eficaz proceso de aprendizaje autorregulado está apoyado en las habilidades pertenecientes tanto a las dimensiones cognitiva y metacognitiva como a la afectivo-motivacional y lo plasman en la siguiente gráfica: 


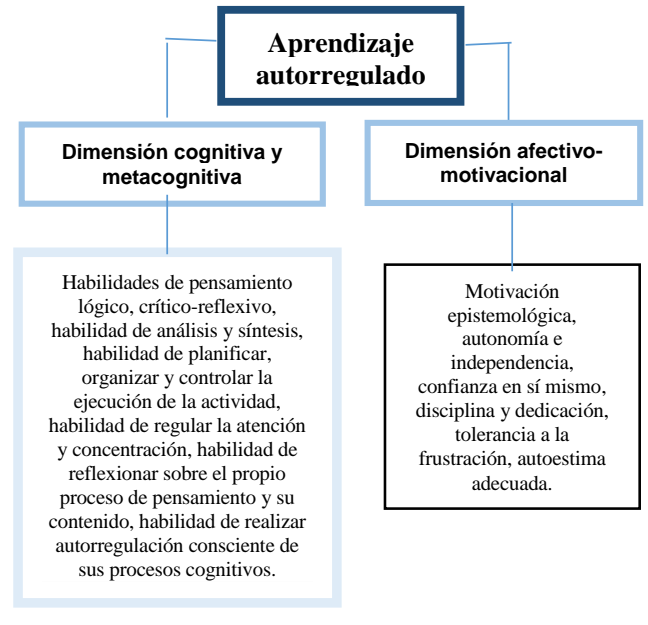

Figura 4. Aprendizaje autorregulado (Klimenko y Alvares, 2009, p.17)

Para efectos de este trabajo, nos enfocaremos en la dimensión cognitiva y metacognitiva, o de reflexión del propio proceso de aprendizaje, cimientos esenciales para el aprendizaje autónomo. Particularmente, mencionaremos como el CAA apoya a los estudiantes a desarrollar la habilidad para analizar sus necesidades, establecer metas, planificar el trabajo y gestionar el tiempo para trabajar. Estas forman parte de una serie de estrategias de aprender a aprender indispensables para los estudiantes que se encuentran en un ambiente de aprendizaje autónomo o semidirigido, en nuestro caso, en el aprendizaje del inglés en el CAA USBI Xalapa.

La función autorreguladora de la metacognición se lleva a cabo a través de la planificación como primer paso. Esta estrategia se realiza antes del inicio de las actividades y ayuda al estudiante a determinar en qué dirección va a dirigirse en su aprendizaje y qué uso hará de las diferentes estrategias: el establecimiento de metas y objetivos; la predicción; la temporalización y asentamiento de un calendario; la decisión de los recursos necesarios para lograr sus metas, así como la selección de materiales y estrategias para llevarlas a cabo.

De acuerdo con Gardner y Miller (2003), la planeación del trabajo es una parte importante en la experiencia de trabajar en un CAA. Se debe apoyar a los estudiantes a ser conscientes de las diversas formas que tienen para planear su trabajo y los asesores deben actuar solo como guías en este proceso y no ser ellos quienes planeen por los estudiantes.

Como parte del proceso de aprendizaje semi-dirigido en los cursos de Inglés I y II del AFBG, los asesores orientamos a los estudiantes para que puedan analizar sus necesidades, establecer sus metas, planificar su trabajo y gestionar el tiempo que requerirán para cubrir este plan de trabajo. Esta guía se inicia desde la primera asesoría del curso y continua a lo largo del curso al comenzar a trabajar cada sección (unidad) que lo componen. Para lograr este fin los asesores diseñamos el formato 'Plan de Trabajo'. Este formato es una especie de contrato de aprendizaje para propiciar y fomentar el aprendizaje autónomo. Lobato describe el contrato de aprendizaje como:

"un acuerdo establecido entre el profesor y el estudiante para la consecución de unos aprendizajes a través de una propuesta de trabajo autónomo, con una supervisión por parte del profesor y durante un período determinado (...) es básico un acuerdo formalizado, una relación de contraprestación recíproca, una implicación personal y un marco temporal de ejecución" (2006, p. 16)

El plan de trabajo, en nuestro CAA, forma parte de un portafolio de evidencias que los estudiantes van creando y organizando a lo largo del curso. Este plan es indispensable para el trabajo que se realizará y debe ser completado por los estudiantes cada vez que inicia las diferentes secciones que integran los módulos de cada taller. Para Inglés I son dos módulos y para Inglés II son tres. Este formato contiene los siguientes elementos: datos, personales, análisis, meta, plan de trabajo y compromiso, como se muestra a continuación:

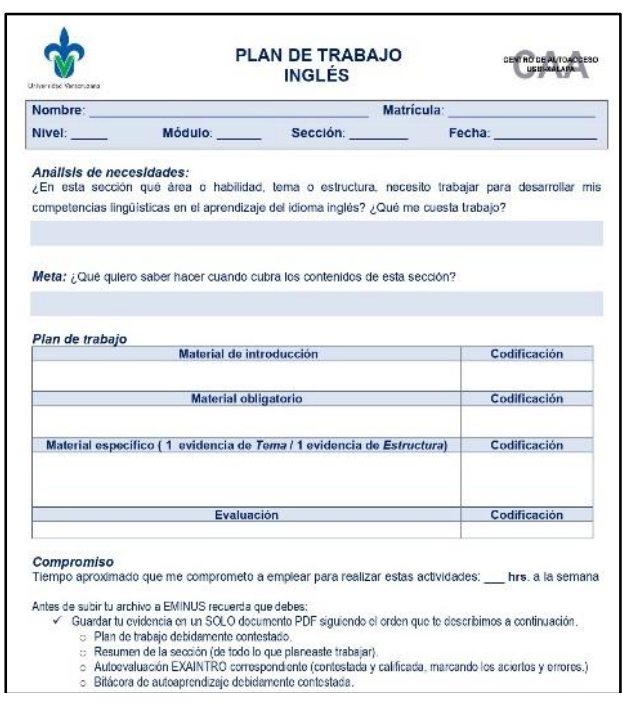

Imagen 3. Formato del Plan de trabajo del CAA USBI Xalapa

Datos personales: Este es el primer apartado del plan donde el estudiante anota su nombre, número de matrícula, de módulo, de sección que va a iniciar y la fecha.

Análisis: En este segundo apartado el estudiante reflexiona sobre lo que se le dificulta o necesita aprender, en este caso, sobre el idioma inglés. Si está llenando este formato por primera vez, se le sugiere que piense en sus experiencias previas, ya sea en sus clases de inglés en la preparatoria o cursos particulares que haya tomado. Sin embargo, si se trata de la segunda vez, o más adelante, puede redactar su análisis con base en la sección que trabajó previamente. Asimismo, se le sugiere que puede incluir información más específica sobre sus necesidades de aprendizaje, por ejemplo, puede anotar alguna habilidad en general: escuchar, hablar, leer, escribir, algún aspecto sobre las reglas gramaticales o vocabulario que desee aprender o reforzar, aspectos de pronunciación, etc. También se le invita a consultar los contenidos lingüísticos que se espera cubra en la sección o los que incluirá en su plan, anotar algún tema o estructura que siempre se les ha dificultado o que crea se le pueda dificultar, para tomar esa información como base para realizar su análisis.

Meta: Su propósito es que el estudiante pueda plantearse objetivos a corto plazo. En este espacio el usuario anota qué es lo que quiere saber hacer al terminar de trabajar la sección que 
está por empezar a planear y llevará a la práctica. Aquí el estudiante debe ser muy específico para que lo que se plantea lograr sea una meta real al concluir su trabajo en la sección. Para poder definir su meta, se le sugiere en primer lugar revisar los contenidos de la sección que va a planear, todos los temas y estructuras en la ruta de trabajo. Igualmente se puede apoyar en la pregunta ¿Qué quiero lograr al finalizar esta sección del curso? y en los ejemplos que le brindamos: Utilizar correctamente el presente continuo; comprender conversaciones donde se intercambie información personal; escribir la descripción de una persona; aprender y utilizar en oraciones vocabulario para describir el clima; comprender textos que hablen de la rutina diaria de las personas, etc.

Plan de trabajo: Este es el aspecto medular del trabajo autónomo que se lleva a cabo en nuestros cursos. En esta parte, los estudiantes con ayuda de la ruta de trabajo (correspondiente al curso al que están inscritos) organizan y eligen los materiales que consideran les ayudarán en su proceso de aprendizaje. Es importante recalcar que debe ser congruente con la meta que establecieron. Como se observa en la figura 7, el plan de trabajo consta de dos columnas, la izquierda destinada a los materiales y la derecha a su codificación. El plan de trabajo tiene la misma distribución de una sección de la ruta de trabajo:

a) Material de introducción: Son llamados INTROS e integran todo el contenido que el estudiante va a trabajar en la sección. Con estos materiales pueden abordar las habilidades receptivas (reading y listening) y productiva de composición (writing). Además, incluyen explicaciones gramáticas y vocabulario en contexto. Están disponibles de manera virtual en la página del CAA y física, en los estantes del centro. Para inglés I existen 12 INTROS y para inglés II, 15.

b) Material obligatorio: Al tratarse de un curso semidirigido, hay ciertos temas que debemos asegurarnos los estudiantes abordarán. Para lograr este objetivo, se incluyen los materiales con el carácter de obligatorio. Estos consisten en aspectos de gramática principalmente, aunque existen materiales que los integran en las diferentes habilidades del idioma.

c) Material específico: Se divide en temas y estructuras. Los temas se refieren a los tópicos que se abordarán en cada una de las secciones, mientras que las estructuras tienen que ver con la gramática. En este apartado es el estudiante quien elige los materiales que trabajará en la sección. Se le sugiere trabajar por lo menos un material de tema y uno de estructura. Igualmente, se le sensibiliza con relación a la importancia de elegir aquellos temas que encuentra difíciles, nuevos o necesita revisar.

d) Evaluación: Para finalizar el trabajo, y a manera de consolidación del mismo, los estudiantes deben responder a una autoevaluación. Esta pequeña prueba aparece al final de cada sección y evalúa aspectos de comprensión auditiva, lectora, composición, gramática y vocabulario. Al tratarse de una autoevaluación, los estudiantes califican dicho examen y reportan su resultado posteriormente.
Compromiso: Para concluir con este plan, el alumno gestiona y acuerda consigo mismo el tiempo que considera que le dedicará a trabajar la sección que está planeando. Este tiempo puede oscilar entre las 3 y 6 horas. Partimos de la premisa de que el usuario se conoce y sabe a qué ritmo trabaja. Se espera también que conforme vaya trabajando en el curso se irá dando cuenta del tiempo que necesita para cada sección e irá anotando un tiempo más real en las siguientes secciones del curso.

Como en cualquier entrenamiento, al inicio del curso la orientación y apoyo para los estudiantes para realizar este proceso es más guiado y preciso. Conforme van avanzando se van volviendo más independientes y sus habilidades metacognitivas se van fortaleciendo hasta el punto de no requerir de nuestra guía para llenar este formato. Este es un ejemplo de un plan de trabajo realizado por un estudiante:

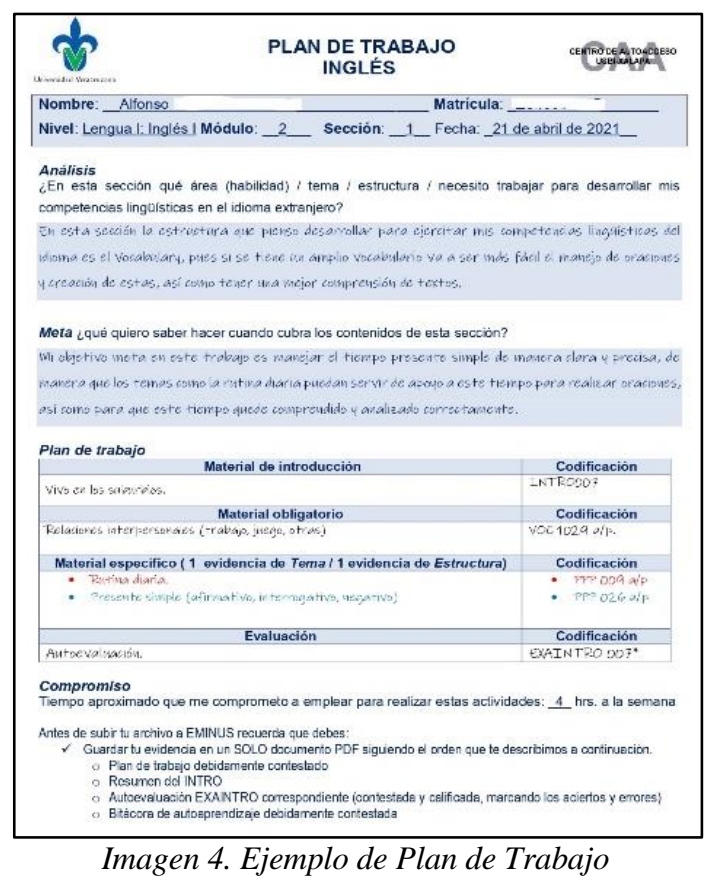

\section{RESULTADOS DE LA DINÁMICA DE TRABAJO SEMIGUIADO EN EL CAA PARA LAS ASIGNATURAS DE INGLÉS I E INGLÉS II}

\section{Estadísticas}

Con el paso de los años, más y más estudiantes han confiado en la modalidad de Autoacceso. Para el año 2017 se contaba con una matrícula de 2153 estudiantes sólo en los cursos de Inglés I e Inglés II. De los estudiantes inscritos más del ochenta por ciento acreditan y generalmente aquellos que cursaron Inglés I optan por continuar con la experiencia educativa Inglés II en esta misma modalidad.

\section{Matrícula del CAA}

Para dar una idea de los índices de aprobación durante los últimos cuatro años, se muestran a continuación algunos números representativos mostrando, el número total de alumnos inscritos, los acreditados y los no acreditados. 
En el año 2017 los números fueron de la siguiente manera:

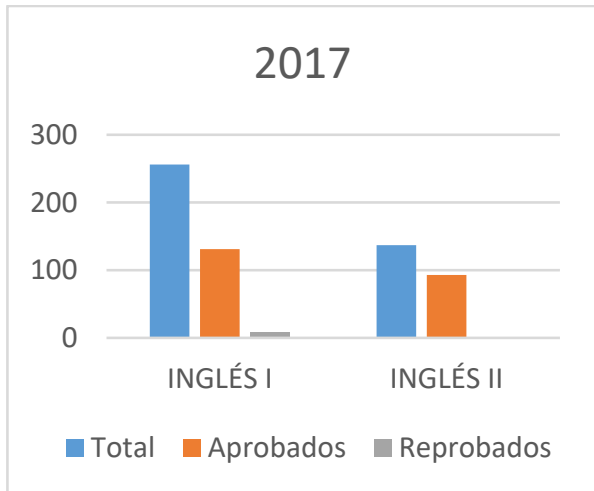

Gráfica 1. Año 2017

Para el año 2018, se notó un incremento en la matrícula como se puede observar:

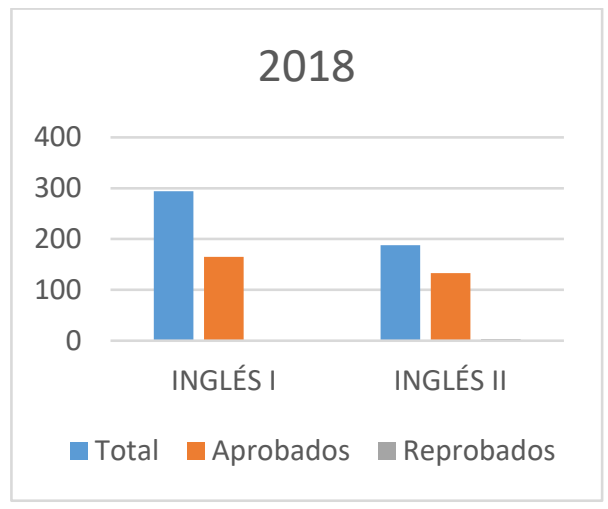

Gráfica 2. Año 2018

En el año 2019, la matrícula de Inglés I aumentó, no así la de Inglés II:

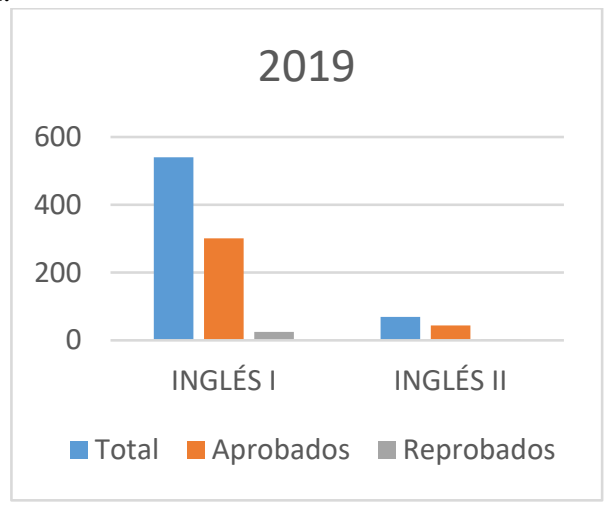

Gráfica 3. Año 2019

En el año 2020, el Centro de Autoacceso USBI Xalapa es reconocido como una entidad académica independiente. En este mismo periodo, a pesar de la situación de contingencia a causa de la pandemia, los números fueron positivos ya que la gran mayoría de los estudiantes acreditaron los cursos. La naturaleza de la dinámica de trabajo y materiales permitieron que estuvieran disponibles de manera virtual en todo momento por lo que los usuarios pudieron trabajar sin problema desde sus lugares de origen.

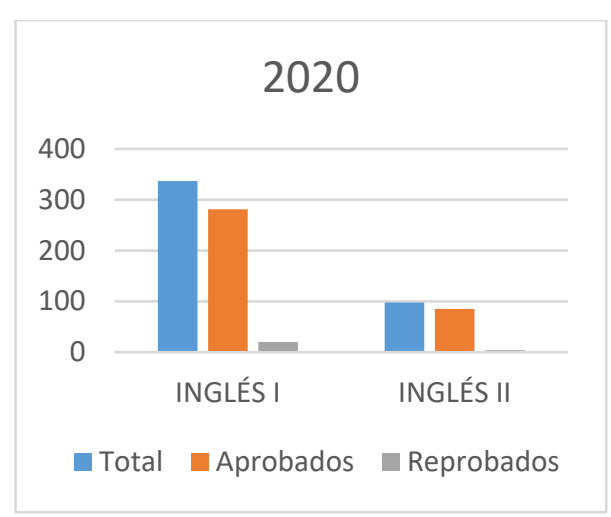

Gráfica 4. Año 2020

Los resultados en el periodo Febrero - Julio 2021 nos muestran que tendríamos una matrícula similar a los periodos anteriores.

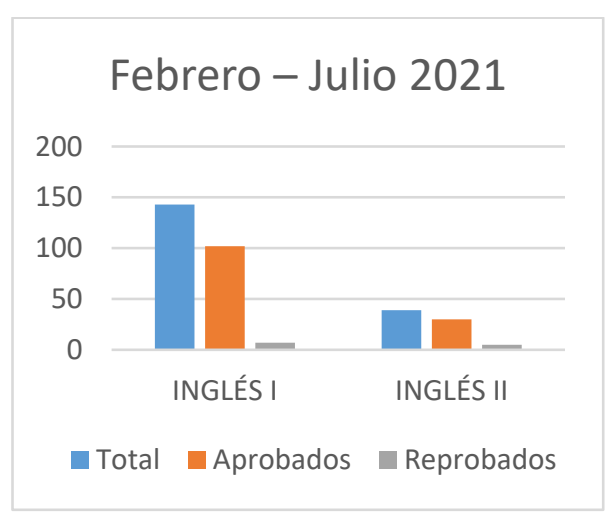

Gráfica 5. Febrero-Julio 2021

Se observa en las gráficas una discrepancia entre el número de acreditados, no acreditados y el total de estudiantes que se debe a que del total de estudiantes con la EE cargada en sus horarios por el sistema, algunos deciden no acudir o cursar estas Experiencias Educativas, por diferentes razones, entre la que destaca el desconocimiento de la dinámica de trabajo. Por esta razón, estos estudiantes terminan con el estatus de "sin derecho". Esto obviamente impacta en nuestros números, sin embargo, del total que cursan la mayoría culminan satisfactoriamente.

\section{Demanda}

Como se mencionó anteriormente la solicitud de cursos autónomos por parte de los estudiantes ha ido en aumento. Cada vez son más los que debido a sus horarios deciden cursar las EE de inglés en el CAA ya que esto representa poder trabajar a su ritmo y en los tiempos que tienen disponibles sin necesidad de desplazarse a las instalaciones todos los días.

Dentro de las carreras que más se atienden en el Centro de Autoacceso se encuentran:

\begin{tabular}{|c|c|c|}
\hline Administración & $\begin{array}{c}\text { Administración de } \\
\text { Negocios } \\
\text { Internacionales }\end{array}$ & Agronomía \\
\hline Artes Visuales & Biología & $\begin{array}{c}\text { Ciencias } \\
\text { Atmosféricas }\end{array}$ \\
\hline Artes Pláticas & Derecho & $\begin{array}{c}\text { Ingeniería } \\
\text { Mecánica Eléctrica }\end{array}$ \\
\hline
\end{tabular}




\begin{tabular}{|c|c|c|}
\hline Ciencias Químicas & $\begin{array}{c}\text { Ingeniería en } \\
\text { Instrumentación } \\
\text { Electrónica } \\
\text { Ingeniería Mecánica }\end{array}$ & $\begin{array}{c}\text { Ingeniería de } \\
\text { Software }\end{array}$ \\
\hline Mutricina & $\begin{array}{c}\text { Química } \\
\text { Farmacéutica } \\
\text { Biológica }\end{array}$ & Ingeniería Química \\
\hline Ingeniería Ambica & Arqueología & Arquitectura \\
\hline Ingeniería Civil & Contaduría & $\begin{array}{c}\text { Ciencias y Técnicas } \\
\text { Estadísticas }\end{array}$ \\
\hline Enfermería & Física & $\begin{array}{c}\text { Gestión y Dirección } \\
\text { De Negocios }\end{array}$ \\
\hline Lengua Francesa & Lengua Inglesa & Matemáticas \\
\hline Pedagogía & $\begin{array}{c}\text { Publicidad y } \\
\text { Relaciones Públicas }\end{array}$ & Psicología \\
\hline $\begin{array}{c}\text { Sistemas } \\
\text { Relaciones } \\
\text { Industriales }\end{array}$ & Administrativos & Teatro \\
\hline
\end{tabular}

\section{REFLEXIONES DE LOS ASESORES}

A lo largo de veinte años de trabajo ininterrumpido, los asesores que iniciamos y los que se han ido integrando al CAA USBI Xalapa, nos hemos percatado de que la práctica de planear el trabajo al inicio de cada sección ha sido de gran beneficio para nuestros estudiantes, ya que además de fomentar el aprendizaje del idioma, involucra el desarrollo de estrategias metacognitivas que les permiten fortalecer el aprendizaje autorregulado y que, además, hoy en día son consideradas habilidades blandas para la vida. Habilidades que complementan tanto su formación académica como personal.

Como lo menciona Guerra (2019) las habilidades blandas son un conjunto tanto de habilidades socio-afectivas como de habilidades para aprender (cognitivas y metacognitivas) y habilidades para el control de la emoción que juntas permiten al individuo a hacer frente a situaciones desafiantes cotidianas y a exigencias del mundo actual. Considerando esta definición, es irrebatible que el fomento de este tipo de destrezas es fundamental en la formación integral de los estudiantes. Esta formación integral es uno de los fines de nuestra universidad y como institución de educación superior, la UV ha implementado acciones para fomentar este tipo de habilidades que al mismo tiempo fortalece uno de sus objetivos principales que es el entrenamiento de competencias duras o habilidades cognitivas, necesarias para el desarrollo de una profesión. Una de estas acciones precisamente ha sido la implementación de Centros de Autoacceso en todas las sedes regionales.

De las destrezas que menciona Guerra (2019) en su definición, en esta reflexión nos estamos enfocando en las habilidades para aprender o metacognitivas. Entre estas habilidades para la vida se pueden mencionar las habilidades para el análisis y solución de problemas, planeación, gestión del tiempo, toma de decisiones, pensamiento crítico, autoevaluación, análisis y comprensión de consecuencias. Sin embargo, nuestras observaciones se dirigen específicamente al análisis de necesidades, establecimiento de metas, la planificación, la organización, la gestión del tiempo y toma de decisiones, ya que son las que han venido desarrollando los estudiantes mediante la realización de su plan de trabajo, según las reflexiones que ellos mismos hacen y que compartiremos más adelante. A continuación, definiremos estos conceptos claves:
Análisis de necesidades: Podríamos definirla como la indagación interna del estudiante sobre las dificultades en su proceso de aprendizaje, en este caso de una lengua extranjera, y en qué situaciones desearía aplicar el conocimiento adquirido. Este análisis está estrechamente relacionado con el siguiente concepto.

Establecimiento de metas: De acuerdo con Castellanos (2010), es una especificación de objetivos basada tanto en las actividades en las que el estudiante podría desear implicarse, así como en el nivel de desarrollo que podría desear alcanzar mediante el uso de la lengua extranjera, es decir, lo que podría desear hacer con las cuatro habilidades.

Planificación: Es la proyección de lo que se va a hacer para lograr las metas establecidas previamente, es decir trazar un plan con las acciones para lograr un fin.

Organización: En una definición amplia, se podría definir como la acción de coordinar, disponer y ordenar los recursos disponibles, ya sean humanos, físicos, etc. y las actividades necesarias para alcanzar los objetivos o metas propuestas.

Gestión o administración del tiempo: Según Reverón (2015), esto se refiere a un proceso en el que se establecen metas $u$ objetivos claros, se deciden las herramientas que facilitan la gestión del tiempo, se tiene en cuenta el tiempo disponible y se verifica el uso que se le da al tiempo, es decir, la percepción del control que se tiene del uso del tiempo personal.

Toma de decisiones: Se puede definir como el proceso de elegir entre las posibles soluciones a un problema y puede darse tanto de forma intuitiva o razonada, o una combinación de ambas.

\section{REFLEXIONES DE LOS ALUMNOS}

Para finalizar, es importante rescatar las reflexiones de los estudiantes sobre el trabajo que se realiza. A través de las actividades finales, los asesores nos hemos percatado que el trabajo de planeación ha favorecido el desarrollo de algunas habilidades blandas que es constatada por los mismos estudiantes en la tarea integradora al final del curso. En esta tarea se les pide hacer una reflexión donde sopesen las actividades llevadas a cabo durante el curso, es decir, sus debilidades, fortalezas y lo que lograron al concluirlo. Es importante enfatizar que son alumnos que cursaron Inglés I o 2 del AFBG en el CAA y para la mayoría fue su primera experiencia de trabajar en modalidad autónoma en su trayectoria escolar. Algunos, de manera general, externan haber adquirido otras habilidades aparte de las linguiísticas para el aprendizaje del idioma:

"Todo genial en el curso, fue una experiencia diferente, se lleva otro ritmo, se aprenden otras habilidades que nos van a servir en otras materias, no solo aprendi el inglés y es bastante agradable trabajar así, me gustaría volver a tomar el siguiente nivel de inglés en próximos semestres". (Carlos)

“..trabajar de esta forma, a veces parece ser un poco más complicado, sin embargo, también te hace desarrollar ciertas destrezas que te sirven en la vida en general, y sobre todo para 
manejar el aprendizaje por cuenta propia, que es como realmente sucede. ;El aprendizaje es libre y está pasando todo el tiempo!" (Melissa)

Otros estudiantes son más específicos en sus reflexiones. Por ejemplo, en los siguientes extractos se deja ver que han podido poner en práctica la habilidad de poder elegir y tomar sus propias decisiones:

"Me ha gustado mucho el poder tener diversas opciones de materiales para escoger y poder estudiar tanto en línea como con la ruta. Pude trabajar solo lo que requería”. (Lilián)

"Considero que el curso es un poco inusual, ya que por lo general estamos acostumbrados a estar en un salón de clases con un maestro que nos enseñe, pero en realidad descubrí que no es difícil a prender por uno mismo y decidir qué trabajar, solo es cuestión de tener disciplina y dedicarle el tiempo necesario". (Ángel)

Algunos aluden a haber desarrollado sus habilidades de planeación y análisis de necesidades, como en este ejemplo:

"A lo largo del curso fui aprendiendo nuevas cosas como habilidades y estrategias, tanto para poder hablar, comprender y escribir en el idioma ingles (sic.) como para planear mi trabajo y pensar lo que me cuesta trabajo" (Alex).

Además, hay quienes externan haberse vuelto más organizados y capaces de gestionar su tiempo para la realización de las actividades:

"Considero que mi desempeño fue satisfactorio, nunca me considere autodidacta pero me asombra lo mucho que aprendí, me volví más organizada y aprendí a manejar mis tiempos."(Tania)

"La ventaja de tomarlo en auto acceso es que se desarrolla esa habilidad de aprender por cuenta propia y tener una mejor disciplina general en todo lo relacionado con lo escolar por ejemplo a organizar mi tiempo y planear lo que tengo que hacer con anticipación”. (David)

Además de externar haber adquirido habilidades que les servirán para su formación académica y personal, varios estudiantes indican de manera general las bondades de trabajar en una modalidad autónoma, tal y como lo vemos en los siguientes fragmentos:

"Esta modalidad de autoaprendizaje es interesante ya que vas a tu paso, eliges que aprender y no es difícil teniendo disciplina." (Pedro).

"Empecé el curso con muchos prejuicios, ideas que me hice a partir de lo que me contaban, que era difícil, fastidioso la modalidad en autoacceso. Sin embargo, me llevé una grata sorpresa al descubrir las grandes ventajas que conlleva, como trabajar a mi propio ritmo, poder acudir en cualquier momento al material disponible, hacerme responsable de mi aprendizaje $y$ contar con alguien que, ante cualquier duda o conflicto, brindará su apoyo." (Caro).
En estas observaciones que incluyen en la conclusión del curso, la mayoría de los estudiantes reconoce que mejoraron sus habilidades lingüísticas del idioma inglés al trabajar en la modalidad autónoma como se muestra en el siguiente ejemplo:

"Realizar esta actividad me hizo darme cuenta que (sic.) durante el curso pude reforzar los conocimientos que había adquirido previamente, lo que me hace sentir bien ya que creo que voy progresando en el idioma inglés. Por lo que mi desempeño en el curso fue aceptable, cumplí con las actividades requeridas y las dudas que iba presentando las iba resolviendo. " (Pedro)

"Al hacer esta actividad pude notar que el vocabulario fue el aspecto en el que tuve más complicaciones, en cuanto a los tiempos y el desarrollo de las oraciones, la dificultad no fue mayor...me siento satisfecho con el trabajo, así como del curso en general, pues pienso que el aprendizaje fue bien logrado, las reglas bien aprendidas, algunas fallas (cosas inevitables) que tendré que practicar más”. (Cristóbal)

\section{CONCLUSIONES}

El Centro de Autoacceso USBI Xalapa se ha caracterizado por ofrecer cursos autónomos a la comunidad universitaria en la región Xalapa por más de veinte años. Durante este periodo ha apoyado al Área de Formación Básica General con cursos de inglés de cuatro habilidades. Para lograr dicho objetivo ha desarrollado una serie de procedimientos, recursos y materiales donde destaca el Plan de Trabajo que es el corazón del trabajo autónomo en estos talleres. Después de varios años de su implementación y actualización se ha identificado que esta planeación no sólo ha ayudado a los estudiantes a desarrollar sus habilidades lingüísticas, si no que ha permeado en otras áreas de su formación. En este sentido, se habla del desarrollo de habilidades blandas para la vida. A través de la observación de los profesores y las reflexiones de los estudiantes constatamos que a través del Plan de Trabajo los estudiantes han podido establecer metas con mayor eficacia, han logrado desarrollar saberes sobre sí mismo y de este modo tomar decisiones sobre su propio aprendizaje, entre otras.

Lo anterior nos deja un buen sabor de boca y nos impulsa a mejorar nuestros procedimientos. Es verdad que la mayoría de nuestros usuarios ven con agrado el trabajo que desempeñamos y acreditan estas experiencias educativas. Sin embargo, todavía existe un número importante de estudiantes que por desconocimiento prefieren no cursar con nosotros, aun cuando están inscritos en los cursos, lo que se ve reflejado en el número de estudiantes que aparecen sin derecho. Esto implica un gran reto que debemos atender en el corto plazo.

Finalmente, nos gustaría señalar que, aunque muchos dudaban de la filosofía de Autoacceso, hoy en día podemos decir que este enfoque ha impactado de manera positiva en muchos de los programas educativos de la Universidad Veracruzana. Un ejemplo de este impacto es que cada vez son más los que han optado por cursos y programas virtuales donde el trabajo se realiza casi en su totalidad de manera autodidacta. De igual manera los ambientes multimodales, donde se mezcla el trabajo guiado con el autoaprendizaje, han cobrado fuerza en nuestra comunidad universitaria. 


\section{REFERENCIAS}

[1] Benson P, Voller P.(1997) Autonomy y independence in language learning. Longman.

[2] Benson, P. (2008). Teachers' and Learners' Perspectives on Autonomy. En: T. Lamb y H. Reinders (Eds.), Learner and Teacher Autonomy: Concepts, Realities and Responses (pp. 1531). Philadelphia: John Benjamins North America

[3] Castellanos I. Análisis de Necesidades y Establecimiento de Objetivos en Didáctica del Español como Lengua Extranjera Expolingua 2002. Monográficos Núm. 10, 2010.Encontrado en: https://marcoele.com/descargas/expolingua_2002.castellanos.p $\underline{\mathrm{df}}$

[4] Dickinson L.(1987). Self-instruction in language learning. Cambridge University Press.

[5] Doddis, A. (1999). David Gardner Y Lindsay Miller. Establishing Self-Access. From Theory to Practice. Lenguas Modernas, 285-291. Recuperado de http://repositorio.uchile.cl/bitstream/handle/2250/139833/E stablishing-self-acccess-From-theory-to-

practice.pdf;jsessionid=9F9B0DBB7885035677A827423A $\underline{881509}$ ? sequence $=1$

[6]Gardner D, Miller L. (2003). Establishing Self-Access. From Theory to Practice. Cambridge University Press. (Original publicado en 1999).

[7] Granados, A. (2017). Mecanismos para estimular el autoaprendizaje. NEXUM; 103(2) 5-7.

[8] Gremmo M y Riley P. (1995) Autonomy, Self-direction and Self-access in Language Teaching and Learning: The History of an Idea. System, 23(2) 151-164.

[9] Guerra S. Una Revisión Panorámica al Entrenamiento de las Habilidades Blandas en Estudiantes Universitarios. Revista Scielo 2019, vol. 23. Corporación Universitaria. Bogotá, Colombia. Encontrado en: https://www.scielo.br/j/pee/a/YyZgKBY9JLVXnCDKMNc $\underline{\text { 7nqc/?lang=es }}$

[10] Harmer, J. (2007). 23 Learner autonomy: learning to learn. The Practice of English Language Teaching. (pp. 394-409). Pearson Longman.

[11] Klimenko O., Alvares J. L. (2009). Aprender cómo aprendo: la enseñanza de estrategias metacognitivas. Educación Y Educadores, 12(2). Recuperado a partir de https://educacionyeducadores.unisabana.edu.co/index.php/eye/ $\underline{\text { article/view/1483 }}$

[12] Lobato C. (2006). Estudio y trabajo autónomo del estudiante, en Mario de Miguel Díaz (coord.) Metodologías de enseñanza y aprendizaje para el desarrollo de competencias, Madrid: Alianza Editorial. Recuperado en https://www.uaem.mx/sites/default/files/facultad-demedicina/descargas/aprendizaje-autodirigido.pdf
[13] Mynard, J. (2018). Chapter 2: Introduction. En C. Ludwig y J. Mynard (Eds.) Autonomy in Language Learning: Advising in Action. (pp. 30-35). Candlin and Mynard.

[14] Olea Deserti, E., Valentín Kajatt, N. O. y Ramírez Martínez, E. T. (2019). La autonomía y el trabajo colaborativo en estudiantes de licenciaturas a distancia en una institución educativa mexicana. 1-17. Recuperado de https://encuentros.virtualeduca.red/storage/ponencias/peru201 9/NEQCEhXBOOZDz8QLQ8Fa0h2SAhL5ozEcekIF10wi.pdf

[15] Peña Calvo, A. (1997). Diseño de Materiales para el Aprendizaje Autónomo de E/LE. p. 621-630. Recuperado de https://cvc.cervantes.es/ensenanza/biblioteca_ele/asele/pdf/08/ 08_0619.pdf

[16] Pozo, J.; Scheuer, N.; Perez, M.; Mateos, M.; Martín, E.; Cruz, M. de la. Nuevas formas de pensar la enseñanza y el aprendizaje. Las concepciones de profesores y alumnos. Barcelona: Editorial Grao, de IRIF, S.L., 2006.

[17] Real Academia Española. (2020). Reproducción. En Diccionario de la lengua española (edición de tricentenario). Consultado el 21 de agosto 2021 de https://dle.rae.es/autonom\%C3\%ADa

[18] Reverón Suárez, N. (2015). La gestión del tiempo. España: Universidad de la Laguna

[19] Serra Salvia, O. (2000). Integrating a self-access system in a language learning institution: a model for implementation. Links y Letters, 95-109. Recuperado de https://raco.cat/index.php/LinksLetters/article/view/22718/225 $\underline{52}$

[20] Sturtridge, G. (1992). Self-Access Preparation and Training. The British Council.

[21] Universidad Veracruzana [UV]. Presentación. Recuperado el 18 de agosto 2021 en: https://www.uv.mx/universidad/presentacion/

[22] Universidad Veracruzana [UV]. Centro de Autoacceso USBI Xalapa. Recuperado el 18 de agosto 2021 en https://www.uv.mx/caausbixalapa/

[23] Universidad Veracruzana [UV]. Dirección de Centros de Idiomas y Autoacceso. Recuperado el 18 de agosto 2021 en https://www.uv.mx/dcia/dcia/antecedentes/

[24] Wenden R, Rubin J. (Eds.). (1987). Learning Strategies in Language Learning. Prentice-Hall International.

[25] Skinner, E. A. y Belmont, M. J. (1993). Motivation in the classroom: Reciprocal Effects of Teacher Behaviour and Student Engagement across the School Year.

[26] Journal of Educational Psychology, 85, 571-581. 